\title{
Combination of coagulant-assisted and SPE purification for the HPLC determination of lycorine and galanthamine in various foods
}

\author{
Jun Sugiura ${ }^{1} \cdot$ Tomoyuki Tsuchiyama $^{1} \cdot$ Masahiro Nakajima $^{1}$
}

Received: 7 April 2019 / Accepted: 24 October 2019 / Published online: 30 October 2019

(c) Springer Nature Switzerland AG 2019

\begin{abstract}
A simple high performance liquid chromatography (HPLC) method has been developed for the analysis of lycorine and galanthamine, typical alkaloids of narcissus, in food. Samples were extracted with sonication using water containing poly-aluminium chloride (PAC) and sodium hydroxide $(\mathrm{NaOH})$ as a solvent followed by solid phase extraction (SPE) with hydrophilic-lipophilic balanced (HLB) cartridge. Elution of the analytes was achieved with $0.1 \mathrm{v} / \mathrm{v} \%$ formic acid kept at $95^{\circ} \mathrm{C}$. Purification with PAC, which is normally used as a coagulant, was successfully combined with SPE, demonstrated the perfect strategy for the removal of interfering substances from the foods. Separation of the analytes was achieved on reversed phase C18 column. The analytes were monitored doubly at wavelengths of 240 and $290 \mathrm{~nm}$ for the detection. The method demonstrated excellent linearity with coefficients of determination $(r(2))$ higher than 0.999 over the range of $0.2-20 \mu \mathrm{g} \mathrm{ml}^{-1}$ for both lycorine and galanthamine. Limits of quantification (LOQs) of the method were in the range of $0.15-0.52 \mathrm{~g} \mathrm{ml}^{-1}$. Method performance was evaluated, demonstrated to have quantitative recoveries of $92.8-98.9 \%$ with satisfactory intra-day precision of lower than $3.7 \%$ and inter-day precision of lower than $5.0 \%$. The method was also applied to a wide variety of foods with complex matrices, indicated the potential of practical use in plant poisoning caused by narcissus contaminated foods.
\end{abstract}

Keywords Lycorine · Galanthamine · Narcissus · Plant poisoning · Poly-aluminium chloride $\cdot$ High performance liquid chromatography (HPLC)

\section{Introduction}

Plant poisoning has been one of the problems confronting Japan in terms of food safety, as vast majority of people enjoy going to natural places such as mountains where a wide variety of plants, both edible and poisonous ones, are grown. Although the number of plant poisoning is not large compared to viral or bacterial food poisonings, they sometimes can be fatal, and should be worth paying attention. Over the past fifty years from 1961 to 2010, growing number of food poisonings caused by higher plants had been reported to Ministry of Health, Labor and Welfare in Japan though the actual number was unknown because of the difference on how to deal with the case depending on times [1]. Among the reported cases, ones caused by narcissus, also called "daffodil" was 31 cases, where a total of 137 people displayed symptoms during the same time period [1]. Plant poisonings caused by narcissus have been observed almost every year, and recently became the most frequently occurred ones of all plant poisonings through 2008 to 2017 [2]. Bulbs and aerial parts of narcissus are frequently mistaken as onions and Chinese chives because of their similarities with these foods in appearance. Plant poisonings caused by narcissus are also seen in other countries. For example, it was reported that a dozen school children in England had been hospitalized after

$\triangle$ Jun Sugiura, vice.city.2016@icloud.com | ${ }^{1}$ Food Department, Nagoya City Public Health Research Institute, 1-11, Hagiyama-cho, Mizuho-ku, Nagoya 467-8615, Japan. 
Fig. 1 Chemical structures of lycorine and galanthamine

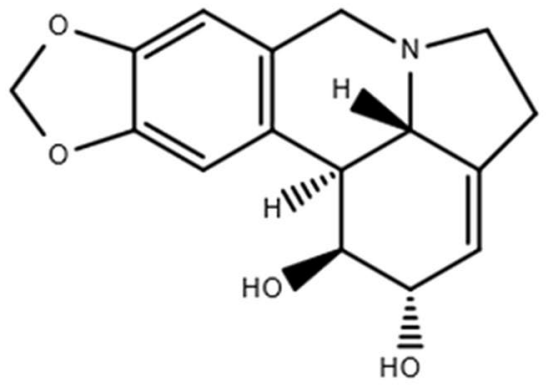

Lycorine

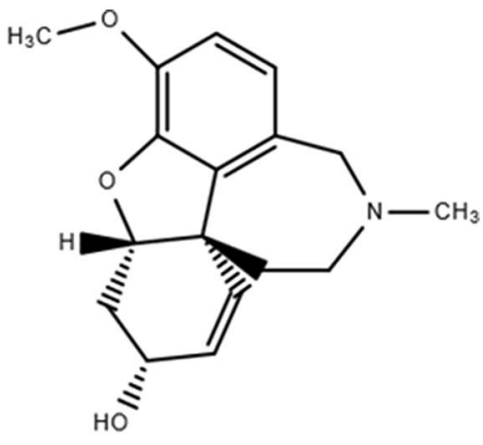

Galanthamine having a soup contaminated with a daffodil bulb in 2009 [3]. It was also a reported case of daffodil ingestion in 2012 that hospitalized 10 people in England [4].

Plant poisoning caused by narcissus usually occurs with symptoms of vomiting, stomachache and diarrhea triggered by alkaloids in the plant [5]. Typical alkaloids found in narcissus are lycorine and galanthamine with the chemical structures shown in Fig. 1. Lycorine have been studied for a long time in search of its beneficial bioactivities, indicated its anti-cancer and anti-virus potentials $[6,7]$, while galanthamine works as an inhibitor of acetylcholine esterase and have been used for the treatment of Alzheimer's disease [8]. Although there are such positive aspects of lycorine and galanthamine, they are also known as alkaloids triggering the symptoms above. In search of the culprit of plant poisoning, analytical method for the detection of alkaloids naturally contained in a certain plant is necessary. Although there are analytical methods developed for the detection of lycorine and galanthamine in the past [9-15], they are all targeted to analytes in plant materials, not intended for food analysis. However, in most cases, narcissus was consumed together with other food as a result of cooking. Thus, analytical method capable of detecting lycorine and galanthamine in food is highly desirable. In our previous study, poly-aluminium chloride (PAC), which is a commonly used coagulant for the purification of water, was used for the clean-up step of HPLC and LC-MS/MS methods for the detection of the both alkaloids from foods [16]. However, the methods developed were specialized only for aerial part of narcissus, and just onestep purification with PAC was considered to be insufficient and cannot be applicable to the food with extremely complex matrices. As a matter of fact, when the method was applied to sauce, which can be used for a wide variety of cuisine, determination of the compounds were practically impossible since interfering peaks were emerged at the same retention time of both lycorine and galanthamine in the chromatogram. For the reasons above, further purification is necessary for applying the method to a wide range of foods. SPE has been one of the most commonly used purification step in sample preparation [17] and considered to be ideal for the further purification because of its applicability in various research fields and availability of a wide variety of packing materials. However, SPE has a shortcoming of having to use large amount of toxic organic solvent such as acetonitrile and methanol in preconditioning and elution steps. For this reason, SPE step with minimized consumption of organic solvent is preferable while maintaining its purification ability. Considering all the reasons above, the purpose of our research is to develop a simple HPLC method with combined purification steps of PAC and SPE with the minimized consumption of organic solvent for the determination of lycorine and galanthamine from various foods.

\section{Materials and methods}

\subsection{Food samples and plant materials}

A total of 6 food samples (curry, stew, miso soup, hashed beef, Japanese-style pancake and Chinese soup) were purchased from local supermarkets in Nagoya, Japan. Ten bulbs of Narcissus tazetta were purchased through the internet and the leaves were harvested at the site of our research institute. Food samples and plant materials were stored at $4{ }^{\circ} \mathrm{C}$ and $-30^{\circ} \mathrm{C}$, respectively until being analyzed.

\subsection{Chemicals and reagents}

Lycorine hydrochloride was purchased from Sigma-Aldrich (MO, USA). Galanthamine hydrobromide was purchased from TOCRIS (Bristol, UK). PAC was purchased from Kishida Chemical. (Osaka, Japan). Sodium hydroxide $(\mathrm{NaOH})$ and formic acid were purchased from Wako Pure Chemical (Osaka, Japan). Acetonitrile and methanol were purchased from Kanto Chemical. (Tokyo, Japan). All reagents used in 
this study were of analytical or HPLC grade. Water used in all experiments was purified through a PURELAB Ultra Analytic system (ORGANO CORPORATION, Tokyo, Japan).

\subsection{Chromatography}

Chromatographic analyses were carried out using prominence HPLC system (Shimadzu, Kyoto, Japan) equipped with solvent delivery unit (LC-20AD), degasser (DGU-20A ${ }_{3}$ ), auto sampler (SIL-20AC HT), column oven (CTO-20AC) and Diode array detector (SPD-M20A). Separation of lycorine and galanthamine was performed on a XBridgeC18 (3.5 $\mu \mathrm{m}$ particle size, $150 \mathrm{~mm}$ length, $4.6 \mathrm{~mm}$ i.d., Waters, MA, USA) using binary linear gradient elution. Two mobile phases were mixed by the binary pump. Mobile phase A: acetonitrile-water-formic acid (50:950:1, v/v/v) and mobile phase B: acetonitrile-formic acid (100:0.1, v/v). Elution program was a liner gradient from $100 \%$ A for the first $4 \mathrm{~min}$, from $100 \mathrm{~A}$ to $0 \% \mathrm{~A}$ in $0.1 \mathrm{~min}$, then held for $2 \mathrm{~min}$, ramped up to $100 \% \mathrm{~A}$ in $0.1 \mathrm{~min}$, held for the final $4.8 \mathrm{~min}$. The total chromatographic run time was $11.00 \mathrm{~min}$. The mobile phase flow rate was $1.0 \mathrm{ml} \mathrm{min}$. The injection volume was $20 \mu \mathrm{L}$. The column oven temperature was set at $40^{\circ} \mathrm{C}$. Detection were performed at wavelengths of $240 \mathrm{~nm}$ and $290 \mathrm{~nm}$.

\subsection{Preparation of standard solution}

Stock standard solutions of lycorine and galanthamine (500 $\mathrm{g} \mathrm{ml}^{-1}$ each) were prepared in methanol, stored at $-20{ }^{\circ} \mathrm{C}$ before use. Working standard solutions ranging from 0.2 to $20 \mathrm{\mu g} \mathrm{ml}^{-1}$ were prepared from stock solution and diluted step by step with water.

\subsection{Sample preparation}

Homogenized sample ( $1 \mathrm{~g}$ ) was accurately weighed into a test tube. Ten $\mathrm{ml}$ water was added to the sample, followed by the addition of $0.5 \mathrm{ml} 30 \mathrm{w} / \mathrm{v} \%$ PAC and $0.5 \mathrm{ml} 10 \mathrm{w} / \mathrm{v} \%$ $\mathrm{NaOH}$. After a brief shake, the solution were sonicated for $30 \mathrm{~min}$. Then, the solution were vacuum filtered through a glass filter into a 20-ml glass beaker, followed by washing with $10 \mathrm{ml}$ water. The filtrate was loaded on to a $60-\mathrm{mg}$ hydrophilic-lipophilic balance (HLB) cartridge from Waters (MA, USA), which was conditioned with $6 \mathrm{ml}$ water. Then, the beaker was washed with $3 \mathrm{ml}$ water and the content was also added to the cartridge. After washing the cartridge with $6 \mathrm{ml}$ water, $5 \mathrm{ml}$ of $0.1 \mathrm{v} / \mathrm{v} \%$ formic acid, which was kept at $95^{\circ} \mathrm{C}$, was instantly applied on the column under vacuum condition. After cooling the obtained solution down to a room temperature, water was added up to $5 \mathrm{ml}$ before HPLC analysis.

\section{Results and discussion}

\subsection{Concentration and volume of $\mathrm{PAC}$ and $\mathrm{NaOH}$ solution}

PAC is widely used for waste water treatment and its effect exceedingly depends on $\mathrm{pH}$ of the solution. Keeping $\mathrm{pH}$ of the solution at an appropriate value is essential to bring out potential of PAC. Our previous study demonstrated that mixing the same amount of $30 \mathrm{w} / \mathrm{v} \% \mathrm{PAC}$ and 10 $\mathrm{w} / \mathrm{v} \% \mathrm{NaOH}$ makes the solution around $\mathrm{pH} 7$ where the effect of PAC become biggest [16]. In the present study, $30 \mathrm{w} / \mathrm{v} \% \mathrm{PAC}$ and $10 \mathrm{w} / \mathrm{v} \% \mathrm{NaOH}$ were similarly used for clean-up process and their amount were set at $0.5 \mathrm{ml}$ each as in our previous study.

\subsection{Selection of wavelength for the detection}

Lycorine and galanthamine are alkaloids and have absorbance peak at the wavelength of $290 \mathrm{~nm}$ as shown in Fig. 2 . In our previous study for lycorine and galanthamine, wavelength of $290 \mathrm{~nm}$ was solely used for the UV detection. The wavelength of $290 \mathrm{~nm}$ is specific and therefore, could alleviate the effect of interfering substances. However, the analysis of standard solution revealed that the wave length of $240 \mathrm{~nm}$ has stronger intensity in terms of signal to noise ratio. For this reason, both wavelengths of 290 and $240 \mathrm{~nm}$ were selected for the detection of lycorine and galanthamine. Monitoring targets at double wavelength was considered to be able to increase the reliability of the result compared to just one wavelength.

\subsection{Selection of SPE cartridge column}

Purification step using PAC can effectively remove interfering substances by taking them into precipitates as a result of coagulation. The PAC purification worked successfully for the determination of artificial sweeteners and preservatives, proved its effectiveness on clean-up process $[17,18]$. However, their concentrations in food are relatively high when compared with potential interfering substances, making them easily detectable under appropriate analytical conditions. However, in plant poisoning cases of narcissus, alkaloid concentration is expected to fluctuate depending on parts of the plant, time and location of harvest and its content in food. Thus, detection of analytes in a wide range of concentration was highly desirable, even in the food with highly complex matrices such as sauce, stew and curry, which can be potential matrices for the analysis of lycorine and galanthamine in food. However, the extract obtained after the PAC purification which we 

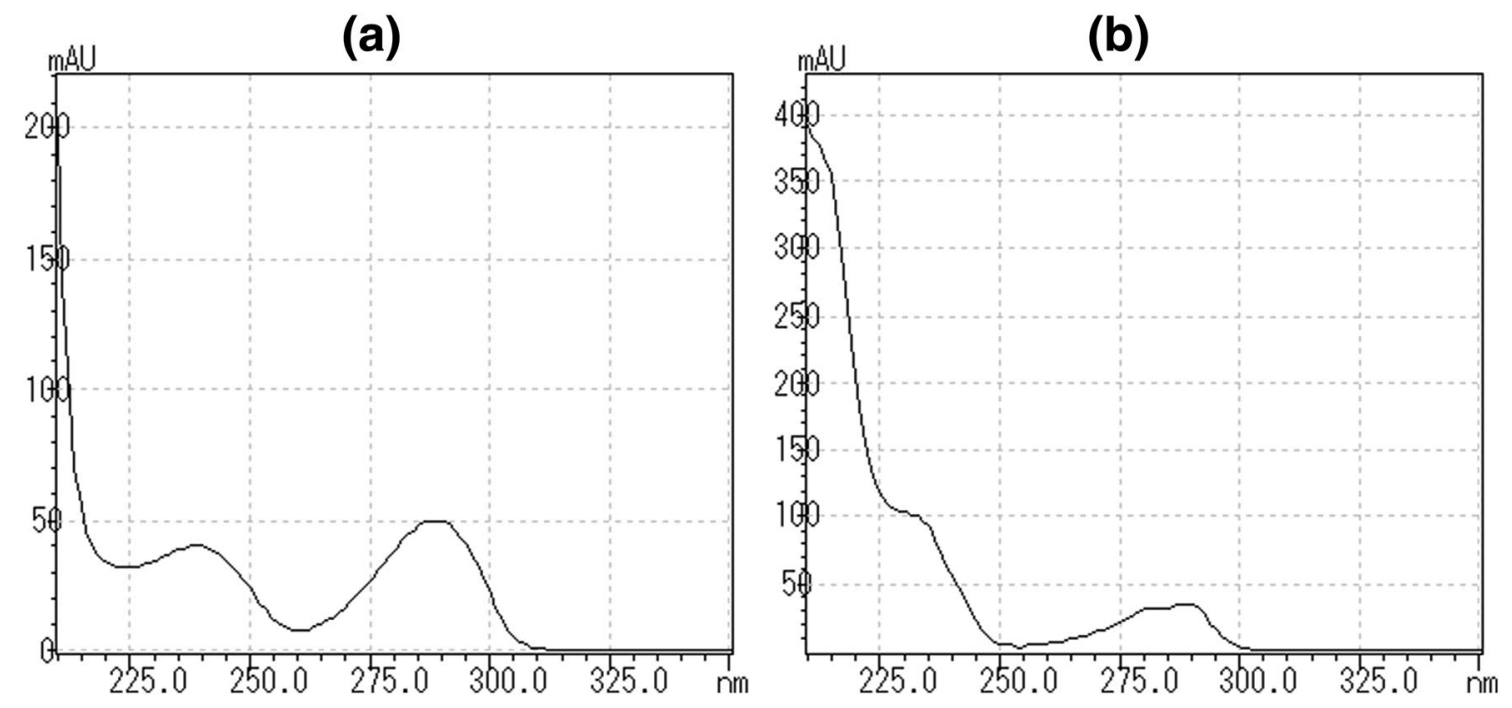

Fig. 2 UV spectra of $\mathbf{a}$ lycorine and $\mathbf{b}$ galanthamine

Fig. 3 Retention behaviour among the five SPE cartirdges

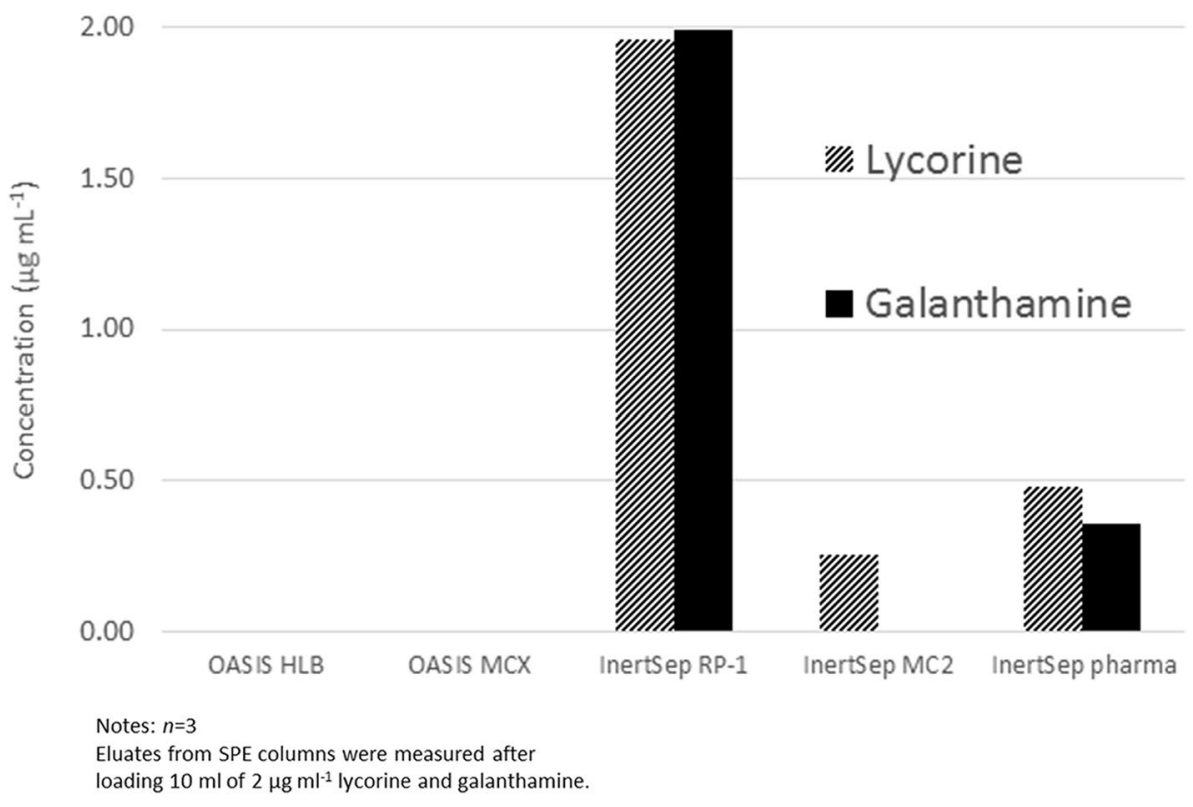

reported on our previous study [16] contains only $0.02 \mathrm{~g}$ sample per milliliter, leading higher limits of detection. Also, when our previously reported method was applied to sauce, interfering peaks were appeared on the chromatogram, made it hard to determining the compounds. What is more, PAC derived metal ions dissolved in the final solution could have negative impact on HPLC column and analytical instrument [17].

For the reasons above, further purification using SPE was considered to be necessary. In this study, five SPE cartridge columns, namely OASIS HLB (Waters, MA, USA), OASIS MCX (Waters, MA, USA), InertSep RP-1 (GL Science, Tokyo, Japan), InertSep pharma (GL Science, Tokyo, Japan) and InertSep MC-2 (GL Science, Tokyo, Japan), were all prepared in a same size $(60-\mathrm{mg}, 3-\mathrm{ml})$ for investigating their retaining capacity of lycorine and galanthamine under a designated condition. Each column was first conditioned with $6 \mathrm{ml}$ of water and loaded with $10 \mathrm{ml}$ of lycorine and galanthamine solution at a concentration of $2 \mu \mathrm{g} \mathrm{ml}^{-1}$. Then, the solution passing through the column were subjected to HPLC analysis for determination. As shown in Fig. 3, both OASIS HLB and OASIS MCX showed strong retention capacity of lycorine and galanthamine as they were not detected from the solution passing through the columns. Tertiary amine in the structure of packing material was considered to play an important role because of 


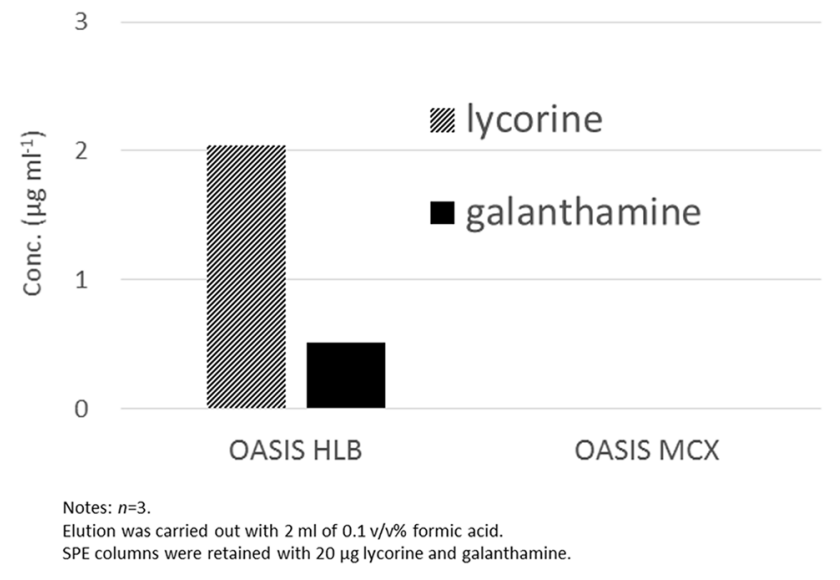

Fig. 4 Elution of lycorine and galanthamine with $0.1 \mathrm{v} / \mathrm{v} \%$ formic acid

the same functional group found in the structures of both lycorine and galanthmaine.

For the elution of lycorine and galanthmaine with solution containing minimum amount of organic solvent, OASIS HLB was suitable because certain amount of both analytes were eluted from the column using $0.1 \mathrm{v} / \mathrm{v} \%$ formic acid as shown in Fig. 4. OASIS MCX still showed strong retention possibly because of the ionic bonding between benzene sulfonate in the SPE column and quaternary ammonium both in lycorine and galanthamine under acidic condition. Although it was possible to elute with organic solvent such as methanol and acetonitrile in basic condition, it's not preferable in terms of the recent trend of green analytical chemistry [19]. For these reasons above, OASIS HLB was finally chosen and used for further study.

\subsection{Elution from SPE cartridge column}

Although $0.1 \mathrm{v} / \mathrm{v} \%$ formic acid has shown its ability to elute analytes from SPE columns, it was necessary to evaluate the $\mathrm{pH}$ of eluting solution as the elution of lycorine and galanthmaine are based on their ratio between ionic and molecular form. Reported pKa of lycorine was 6.4 [20], while that of galanthamine was reported to be 8.2 [21]. For determining the appropriate concentration of the eluting solution, the $\mathrm{pH}$ measurement of formic acid at concentrations of $0.01,0.1$ and $1 \mathrm{v} / \mathrm{v} \%$ were carried out with a $\mathrm{pH}$ meter, indicated that their $\mathrm{pH}$ values were 3.3, 2.7 and 2.2, respectively. All the $\mathrm{pH}$ values measured were well below the pKa of lycorine and galanthamine, meaning the majority of analytes are existing as ionic form which has weaker interactions with column packing material. However, a concentration of $0.1 \mathrm{v} / \mathrm{v} \%$ was thought to be appropriate and chosen because of the same concentration of HPLC mobile phase.
As speculated from Fig. 4, the total volume of the solution necessary for complete elution was considered to be large, which could decrease the sensitivity of the method. In hope of minimizing the solution needed for the elution, enhancing its elution strength by adjusting the temperature of the solution was thought to be ideal because of unnecessity to add extra organic solvent to the solution. For the purpose of assessing the temperature effect on elution strength, temperatures of $20,50,70$ and $95^{\circ} \mathrm{C}$ were investigated by fractionally analyzing eluates from SPE columns. For maintaining the designated temperatures above, the solution was kept in a water bath with a thermometer. Handlings on the experiments were carried out in a prompt way for the prevention of temperature drop of the solution while in operation. Vacuum strength in a manifold was also increased for the same purpose. As can be seen in Fig. 5, elution strength dramatically increased along with the temperature rise. Temperatures of $50{ }^{\circ} \mathrm{C}$ seemed to be enough for the complete elution of lycorine, but large amount of galanthamine still remained on the column. On the other hand, the highest temperature of $95^{\circ} \mathrm{C}$ showed the ideal elution pattern because over $95 \%$ of lycorine and galanthamine were eluted within $5 \mathrm{ml}$. Therefore, in further study, the eluting solution of $0.1 \mathrm{v} / \mathrm{v} \%$ formic acid was kept at a temperature of $95^{\circ} \mathrm{C}$ before the elution.

\subsection{Influence of sonication time}

For the optimization of sonication time, extraction efficiency was investigated using both bulb and aerial of narcissus. Homogenized sample $(1 \mathrm{~g})$ was weighed into a test tube followed by the addition of $10 \mathrm{ml}$ water, $0.5 \mathrm{ml} 30$ $\mathrm{w} / \mathrm{v} \% \mathrm{PAC}$ and $0.5 \mathrm{ml} 10 \mathrm{w} / \mathrm{v} \% \mathrm{NaOH}$ solutions. Then, the tube was sonicated for a designated duration. After that, solution in the tube was vacuum filtered followed by washing with $10 \mathrm{ml}$ water. Finally, the solution was filled up to $20 \mathrm{ml}$ with water before HPLC analysis. Sonication times of $0,5,10,30$ and 50 min were applied to the bulbs and that of 0,10 and 30 min were applied to the leaves. At the same time, an extraction method with methanol and homogenization [12] with slight modification was also performed for comparing the efficacy of extraction between the two methods. Briefly, $1 \mathrm{~g}$ sample was added with $25 \mathrm{ml}$ methanol followed by homogenization (7500 rpm, $5 \mathrm{~min}$ ) and vacuum filtration. Then, filter was washed three times with $25 \mathrm{ml}$ methanol each and finally, the solution was evaporated to dryness followed by reconstituting with $20 \mathrm{ml}$ water. As can be seen in Fig. 6, concentrations of lycorine and garanthamine were increased along with the time of sonication in bulb and reached to the level obtained from the other method at around $30 \mathrm{~min}$ of sonication. On the other hand, concentration of lycorine was at a standstill 
Fig. 5 Elution of analytes with $0.1 \mathrm{v} / \mathrm{v} \%$ formic acid in various temperature
15

\section{Lycorine}

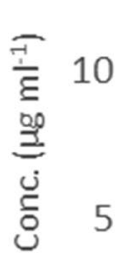

$\equiv 70^{\circ} \mathrm{C} \quad \% 95^{\circ} \mathrm{C}$

0

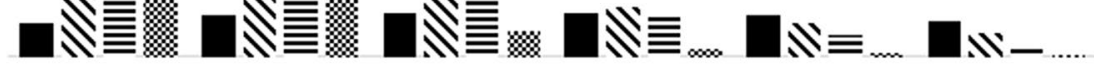

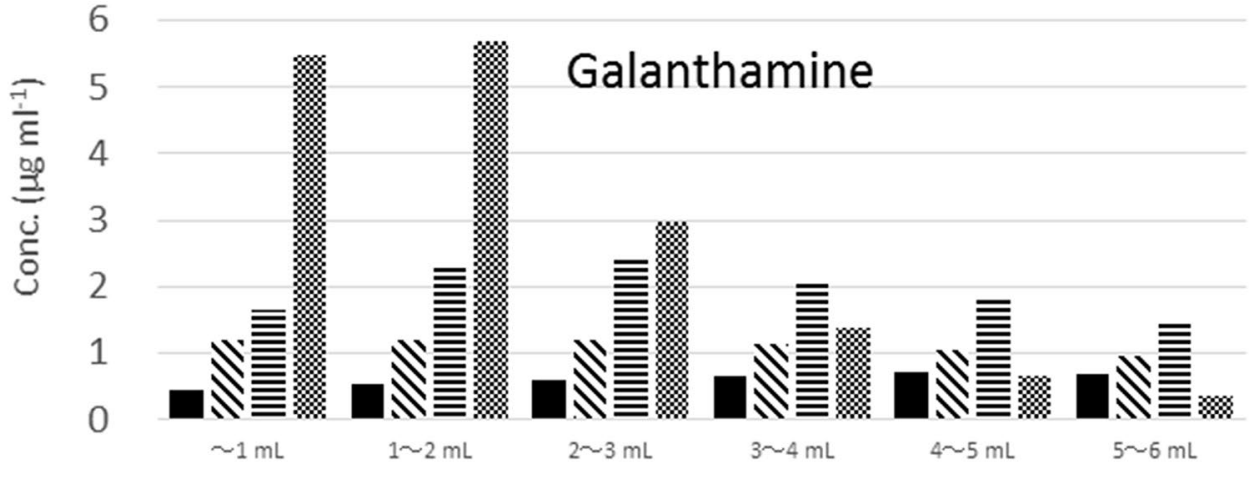

Notes: $n=3$.

Lycorine and galanthamine were fractionally eluted with $0.1 \mathrm{v} / \mathrm{v} \%$ formic acid.

SPE columns were retained with $15 \mu \mathrm{g}$ lycorine and galanthamine.

$0.1 \mathrm{v} / \mathrm{v} \%$ formic acid was kept in water at a fixed temperature. (a)

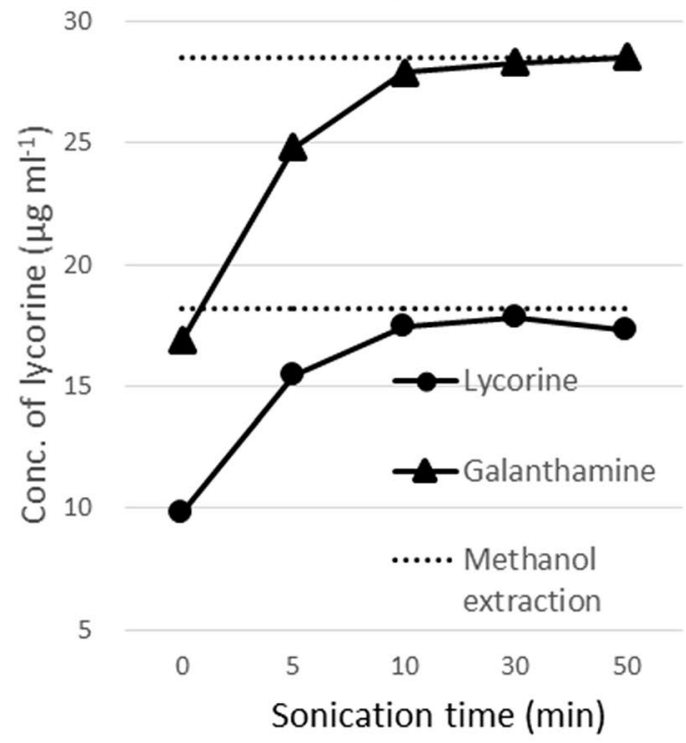

(b)

1.2

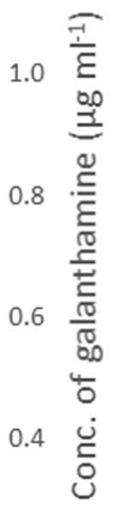

0.2
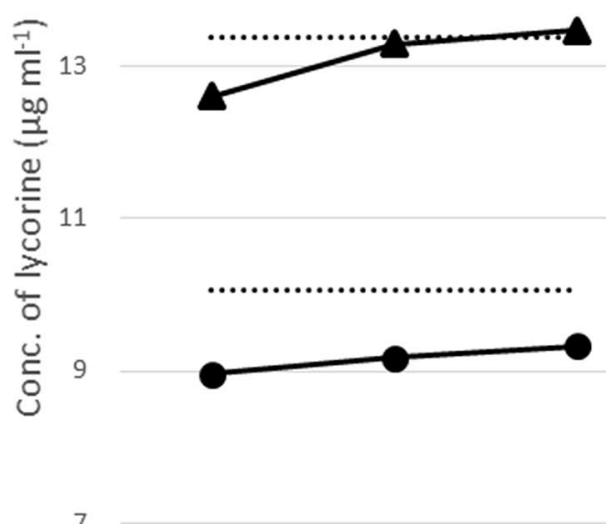

0.9

0.9

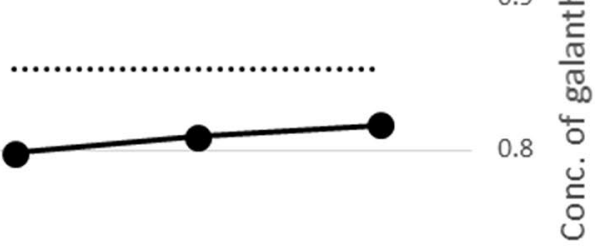

0.8

Notes: $n=3$.

$1 \mathrm{~g}$ sample was added with $10 \mathrm{ml}$ water followed by the addition of polyaluminium chloride (PAC) and $\mathrm{NaOH}$ solutions before sonication.

Fig. 6 Extraction from $\mathbf{a}$ a bulb and $\mathbf{b}$ leaves of narcissus with different time of sonication and comparison with the methanol extraction method 
Fig. 7 Chromatograms of narcissus leaf extract after performing (a) the proposed extraction and (b) the methanol extraction

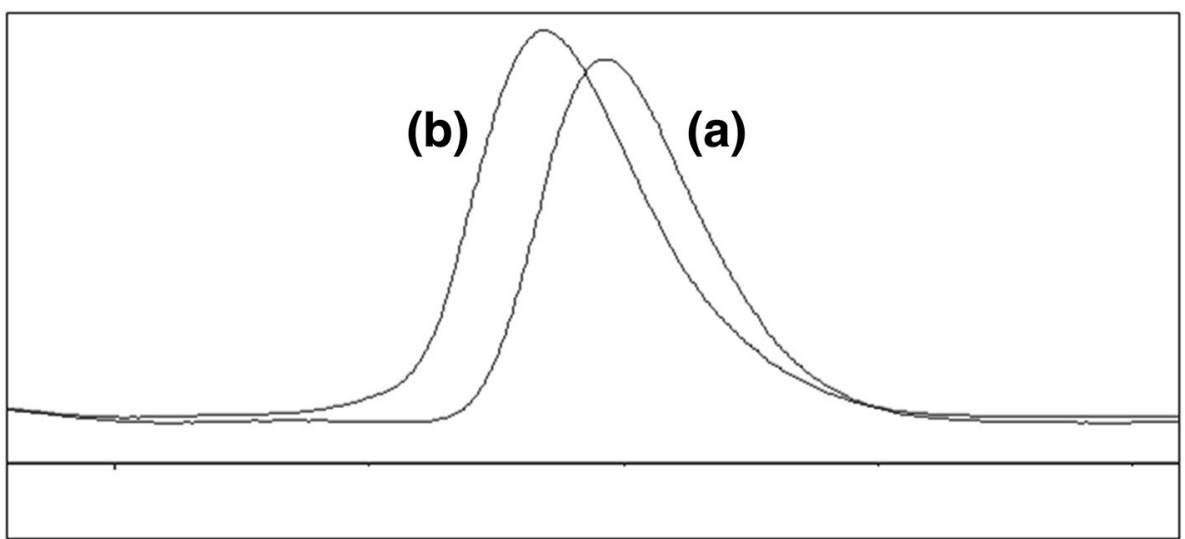

Notes: Sonication time was $30 \mathrm{~min}$ for the proposed extraction. Detection wavelength was set at $240 \mathrm{~nm}$. and slight below the level of the other method even in the 30 min of sonication. A possible reason for the gap of concentration between the two methods could be explained by the existence of interfering peak appearing at almost the same retention time as lycorine. When comparing the chromatograms between the two methods, lycorine peak in the chromatogram obtained from the methanol extraction had wider peak width than the other as in Fig. 7. Spectral patterns at the both front and rear edges of the peak were different from the standard spectrum of lycorine, indicating the presence of interference over lycorine peak. Judging from the reason above-mentioned, lycorine in leaves was also considered to be sufficiently extracted with 30 min of sonication and therefore, sonication time was set at 30 min for further study.

\subsection{Effect of each purification step}

To confirm the necessity and effect of each purification step, experiments had to be carried out in a sample with complex matrices. As a typical sample with highly complex matrices, sauce was selected for the study because it is a mixture of a wide variety of food with dark color, making it relatively easy to visualize the deference in effect among each purification step. More importantly, sauce could be potential matrix for narcissus' food poisoning because of its wide applicability to cooked food. There were three patterns investigated and the first one was water extraction only. In the water extraction, $1 \mathrm{~g}$ sample was added with $10 \mathrm{ml}$ water followed by $30 \mathrm{~min}$ of sonication. Then, the solution was vacuum filtered with a glass filter, finally filled up to $20 \mathrm{ml}$ with water. The second one was extraction using PAC. In the second pattern, $0.5 \mathrm{ml} 30 \mathrm{w} / \mathrm{v} \% \mathrm{PAC}$ and $0.5 \mathrm{ml} 10 \mathrm{w} / \mathrm{v} \% \mathrm{NaOH}$ were added after $10 \mathrm{ml}$ water addition. The last one was extraction using PAC followed by SPE clean-up, the proposed method. In the last one, the solution obtained in the second pattern were subjected to the optimized SPE process. But the final solution were filled up to $20 \mathrm{ml}$ with water for making sample concentration in the final solution even. Visual investigation between the first and second patterns revealed that PAC coagulation perfectly worked for the removal of pigments as the first and second patterns yielded blown colored and transparent solutions, respectively. As can be seen in Fig. 8, even only the PAC purification made a deference in the chromatogram when compared with the water extraction. Specifically, the removal of interfering substances in an early retention time was successfully achieved. However, there were still interfering peaks potentially becoming obstacle for the determination of lycorine and galanthamine. After additional purification step with SPE, the interfering peaks were completely disappeared. Although water extraction followed by SPE purification also demonstrated huge effect in the chromatogram, loss of lycorine from the SPE column and a minor interfering peak over galanthamine's were observed as in Fig. 9. The recovery loss was possibly caused by acid condition of the extract, weakening the affinity of lycorine to the solid phase because of the surge of ionic form. On the other hand, PAC extract could maintain its $\mathrm{pH}$ value neutral, indicating successful removal of acids from samples. The PAC clean-up process was also preferable in a way that it could cut the time of filtration by almost half compared with the water extraction. It was speculated that particles in the water extract are small enough to be caught in a filter network, gradually accumulated to the level in which water cannot easily pass through. In contrast, the PAC extract forms aggregation of particles large enough to be on top of the filter paper, making it easier for water penetration through it.

In this study, once again, the PAC extraction followed by SPE clean-up demonstrated its necessity and effectiveness. 
(a)

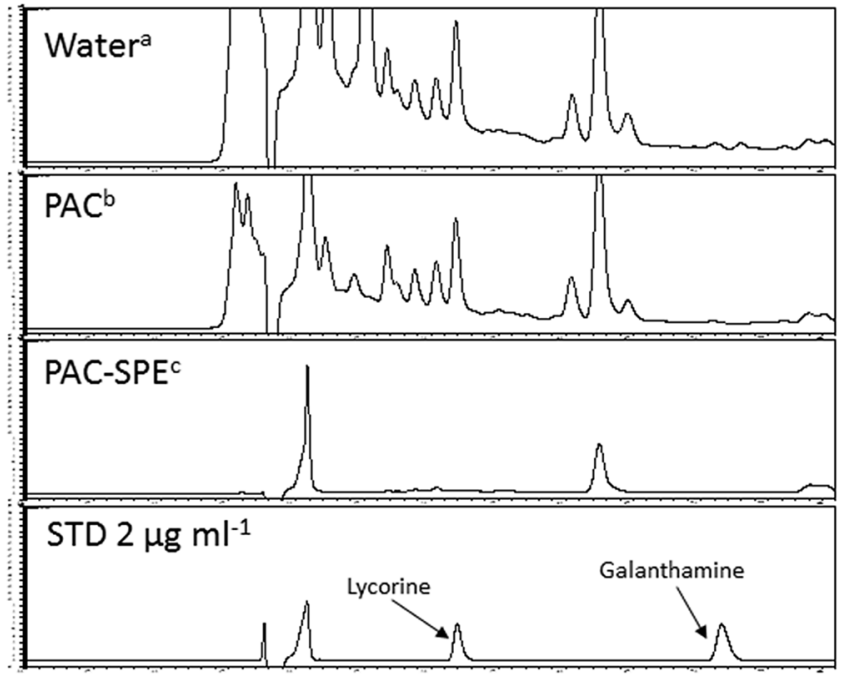

(b)

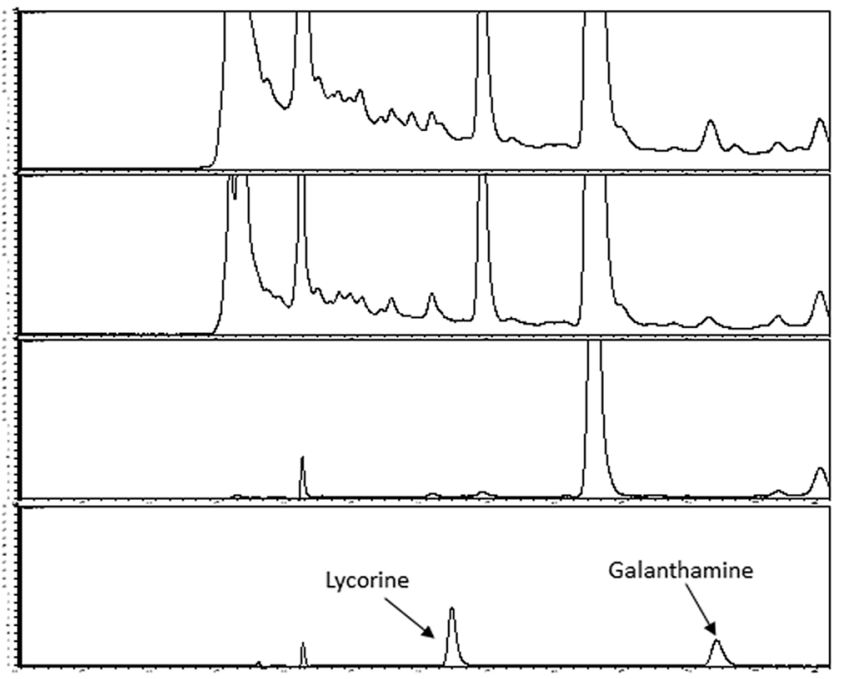

Notes: Sauce was used as a sample and finally filled up to $20 \mathrm{ml}$ with water after purification.

a Sample was added with $10 \mathrm{ml}$ water before extraction.

${ }^{b}$ Sample was added with $10 \mathrm{ml}$ water followed by addition of poly-aluminium chloride (PAC) and $\mathrm{NaOH}$ solutions.

'Solution obtained from the PAC extraction was subjected to SPE purification.

Fig. 8 Effect of each purification step on chromatogram at wavelength of a $240 \mathrm{~nm}$ and b $290 \mathrm{~nm}$
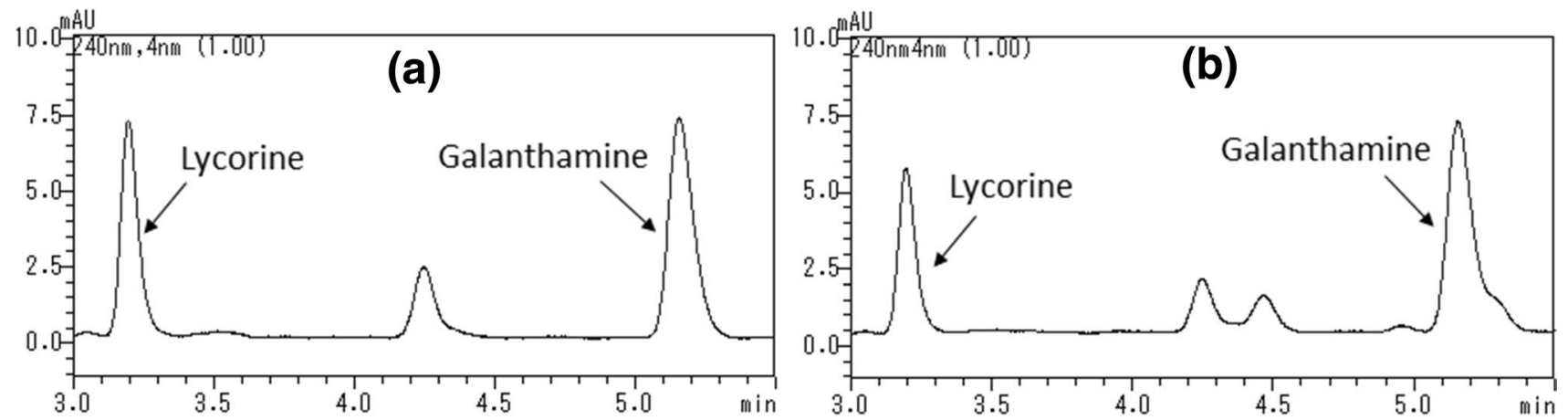

Notes: Sauce was used as a sample and spiked with $50 \mu \mathrm{g}$ lycorine and galanthamine.

Wavelength of $240 \mathrm{~nm}$ was used for detection.

a Sample was extracted with $10 \mathrm{ml}$ water followed by the optimized SPE purification.

Fig. 9 Difference on chromatograms of sample solution processed with $\mathbf{a}$ the proposed method and $\mathbf{b}$ water extraction-SPE

\subsection{Method validation}

The method validation, including linearity, LOQs, recovery and precision was carried out. Recovery and precision tests were performed using sauce as a sample in duplicate successively for five days. Spiked levels of the sample were 5 and $50 \mu \mathrm{g} \mathrm{g}^{-1}$. Although a study reported the concentrations of lycorine and galanthamin are varying from 49.2 to $462 \mu \mathrm{g} \mathrm{g}^{-1}$ and from 12.0 to $70.6 \mu \mathrm{g} \mathrm{g}^{-1}$, respectively in bulb [12], the selected spiked concentrations were determined after considering the degree of dilution through cooking process. As in Table 1, result of the experiments were satisfactory, demonstrating the recoveries ranging from 92.8 to $98.9 \%$ with intra-day precision of less than $3.7 \%$ and inter-day precision of less than $5.0 \%$. Linearity was tested by injecting standard solutions containing varying levels of the target compounds. In both lycorine and galanthamine, at both wavelengths of $240 \mathrm{~nm}$ and $290 \mathrm{~nm}$, excellent linearity and coefficients of determination over 0.999 were obtained in the range of 0.2 to $20 \mathrm{\mu g} \mathrm{ml}^{-1}$. The LOQs of the method were evaluated by continuously injecting standard solution of $0.2 \mathrm{\mu g} \mathrm{ml}^{-1}$ for 
Table 1 Repeatability and reproducibility of the method at two spiked levels

\begin{tabular}{llclll}
\hline $\begin{array}{l}\text { Detection wave- } \\
\text { length }(\mathrm{nm})\end{array}$ & Analyte & $\begin{array}{l}\text { Spiked level } \\
(\mu \mathrm{g})\end{array}$ & $\begin{array}{l}\text { Mean recov- } \\
\text { ery }(\%)\end{array}$ & $\begin{array}{l}\text { Intra-day preci- } \\
\text { sion }(\%)\end{array}$ & $\begin{array}{l}\text { Inter-day } \\
\text { precision } \\
(\%)\end{array}$ \\
\hline 240 & Lycorine & 5 & 98.4 & 3.7 & 5.0 \\
& & 50 & 98.5 & 1.6 & 2.1 \\
& Galanthamine & 5 & 92.8 & 2.6 & 2.6 \\
& & 50 & 98.9 & 2.1 & 2.1 \\
& Lycorine & 5 & 97.2 & 2.8 & 2.9 \\
& & 50 & 96.8 & 1.8 & 1.9 \\
& Galanthamine & 5 & 96.5 & 2.0 & 2.0 \\
& & 50 & 97.7 & 2.3 & 2.3 \\
\hline
\end{tabular}

Sauce was used as a sample

Experiments were performed in duplicate successively for five days
Table 2 Result of the spike and recovery tests on six foods

\begin{tabular}{|c|c|c|c|c|}
\hline & \multicolumn{2}{|l|}{ Lycorine } & \multicolumn{2}{|c|}{ Galanthamine } \\
\hline & $\begin{array}{l}\text { Mean } \\
\text { recovery } \\
(\%)\end{array}$ & RSD (\%) & $\begin{array}{l}\text { Mean } \\
\text { recovery } \\
(\%)\end{array}$ & RSD (\%) \\
\hline Curry & 99.8 & 4.0 & 102.4 & 3.1 \\
\hline Stew & 101.5 & 0.2 & 95.5 & 1.3 \\
\hline Miso soup & 105.0 & 1.5 & 103.4 & 1.2 \\
\hline Hashed beef & 105.1 & 2.3 & 93.2 & 3.4 \\
\hline Japanese-style pancake & 102.9 & 0.8 & 95.7 & 1.9 \\
\hline Chinese soup & 103.4 & 1.3 & 100.0 & 1.9 \\
\hline
\end{tabular}

$n=3$

$1 \mathrm{~g}$ sample was spliked with $5 \mu \mathrm{g}$ lycorine and galanthamine

twenty times. Then, concentrations equivalent to 10 times of standard deviation were calculated. As a result, LOQs of lycorine and galanthamine at $290 \mathrm{~nm}$ were 0.26 and $0.52 \mu \mathrm{g} \mathrm{g}^{-1}$, respectively and that at $240 \mathrm{~nm}$ were 0.15 and $0.27 \mu \mathrm{g} \mathrm{g}^{-1}$, respectively.

\subsection{Application of the method to various foods}

To confirm the applicability of the method to a wide variety of foods, six foods were prepared and analyzed with the proposed method. Foods prepared were curry, stew, miso soup, hashed beef, Japanese-style pancake and Chinese soup, all considered to have a variation of matrices and to be potential cause of narcissus' food poisoning. Every food was divided into two portions. One was spiked with $5 \mu \mathrm{g}$ lycorine and galanthamine for performing spiked and recovery tests and the investigation of interfering originated from the food. The recovery tests were performed in triplicate. The other portion was intentionally contaminated with narcissus for producing the actual situation of food poisoning. As shown in Table 2, results of spiked and
Table 3 Result of the tests on foods contaminated with narcissus

\begin{tabular}{lllllll}
\hline & \multicolumn{2}{l}{ Lycorine } & & & \multicolumn{2}{l}{ Galanthamine } \\
\cline { 2 - 3 } & Conc. $\left(\mu \mathrm{g} \mathrm{g}^{-1}\right)$ & S.D. & & Conc. $\left(\mu \mathrm{g} \mathrm{g}^{-1}\right)$ & S.D. \\
\hline Curry & 57.4 & 0.67 & 3.51 & 0.07 \\
Stew & 80.2 & 0.85 & 5.24 & 0.11 \\
Miso soup & 66.0 & 0.57 & 3.80 & 0.08 \\
Hashed beef & 80.7 & 0.99 & 3.68 & 0.02 \\
Japanese-style pan- & 19.5 & 0.17 & 1.98 & 0.05 \\
$\quad$ Cake & & & & & \\
Chinese soup & 13.9 & 0.12 & & 1.15 & 0.03
\end{tabular}

$n=3$

Curry, stew, miso soup and hashed beef were homogenized together with a bulb of narcissus

Japanese-style pancake and Chinese soup were homogenized together with leaves of narcissus

recovery tests were satisfactory, demonstrating the recovery range of $99.8-105.1 \%$ for lycorine and $93.2-103.4 \%$ for galanthamine. As shown in Table 3, result of analysis of the foods contaminated with narcissus was also successful of being able to detect both analytes in all foods, proving the method applicability on actual contamination. The detected levels of lycorine and galanthamine were ranging $13.9-80.7 \mu \mathrm{g} / \mathrm{g}$ and $1.15-5.24 \mu \mathrm{g} / \mathrm{g}$, respectively. Though minimal dose of lycorine and galanthamine that cause the symptoms to human is still not clearly known, there is a study experimenting effects of lycorine in beagle dogs, proved that emesis starts at $0.5 \mathrm{mg} / \mathrm{kg}$ [22]. The tendency is not directly applicable to human, however, the detected levels can be toxic depending on the amount of ingestion. More importantly, there are a variety of alkaloids found in narcissus [23] except for lycorine and galanthamine, indicating that much smaller amount can cause serious symptoms. 


\section{Conclusions}

The proposed method demonstrated successful combination of PAC-assisted and SPE purification for the removal of interfering substances while concentrating lycorine and galanthamine. The method also took advantage of hot water in SPE elution, successfully minimizing the consumption of organic solvent. Satisfactory results for linearity, LOQs, recovery and precision were obtained on the developed method. Furthermore, applicability of the method to a wide variety of foods was also demonstrated. The method showed its possible usage on actual food poisoning or accidental ingestion of narcissus which is one of the most frequent cause of plant poisoning in Japan. It's especially useful in case where there are only processed samples left, contributing the accurate determination of the target compounds and the faster result of cause investigation.

\section{Compliance with ethical standards}

Conflict of interest The authors declare that they have no conflict of interest.

\section{References}

1. Toda M, Uneyama C, Kasuga $F$ (2014) Trends of plant toxin food poisonings during the past 50 years in Japan. Food Hygiene Saf Sci 55:55-63

2. [MHLW] Ministry of Health, Labour and Welfare (2018) [internet]. [cited 2018 Apr 16]. http://www.mhlw.go.jp/stf/seisakunitsuite/ bunya/kenkou_iryou/shokuhin/yuudoku/index.html

3. [BBC NEWS 2009] British Broadcasting Corporation NEWS (2009) [internet]. [cited $2018 \mathrm{Apr} 16$ ]. http://news.bbc.co.uk/2/hi/uk_ news/england/suffolk/8031344.stm

4. [BBC NEWS 2012] British Broadcasting Corporation NEWS (2012) [internet]. [cited 2018 Apr 16]. http://www.bbc.com/news/ukengland-bristol-17233766

5. Kasahara Y (2010) A recent tendency of food poisoning caused by toxic plants and its problem awaiting solution. Food Hygiene Saf Sci 51:311-318

6. Wang P, Li L, Wang Q, Shang L, Shi P, Yin Z (2014) Anti-denguevirus activity and structure-activity relationship studies of lycorine derivatives. ChemMedChem 9:1522-1533

7. Li X, Xu P, Wang C, Xu N, Xu A, Xu Y, Sadahira T, Araki M, Wada K, Matsuura E, Watanabe $M$, Zheng J, Sun P, Huang P, Nasu Y, Liu C (2017) Synergistic effects of the immune checkpoint inhibitor CTLA-4 combined with the growth inhibitor lycorine in a mouse model of renal cell carcinoma. Oncotarget 8:21177-21186

8. Blesa R, Davidson M, Kurz A, Reichman W, Baelen B, Schwalen S (2003) Galantamine provides sustained benefits in patients with 'advanced moderate' Alzheimer's disease for at least 12 months. Dement Geriatr Cogn Disord 15:79-87
9. Davey M, Persiau G, Bruyn A, Damme J, Bauw G, Montagu M (1998) Purification of the alkaloid lycorine and simultaneous analysis of ascorbic acid and lycorine by micellar electrokinetic capillary chromatography. Anal Biochem 258:80-88

10. Abou-Dania A, Toaima S, Hammoda H, Shawky E (2007) New rapid validated HPTLC method for the determination of lycorine in amaryllidaceae plants extracts. Chromatographia 65:497-500

11. Mroczek T, Mazurek J (2009) Pressurized liquid extraction and anticholinesterase activity-based thin-layer chromatography with bioautography of Amaryllidaceae alkaloids. Anal Chim Acta 633:188-196

12. Shimodozono E, Iwaya A, Enomoto K, Fukushiyama I, Yoshimura K (2012) Analysis of lycorine and galanthamine in amaryllidaceae. Annu Rep Kagoshima Prefect Inst Environ Res Public Health. 13:80-83

13. Chun J, Jang I, Arasu M, Al-Dhabi N, Duraipandiyan V, Lee D, Lee S, Kim S (2013) Isolation and identification of alkaloids and anthocyanins from flower and bulb of Lycoris radiata using HPLC and LC-ESI-MS. J Agric Chem Environ 2:22-26

14. Kaya G, Cicek Polat D, Emir A, Bozkurt Sarikaya B, Onur M, Unver Somer N (2014) Quantitative determination of galanthamine and lycorine in Galanthus elwesii by HPLC-DAD. Turk J Pharm Sci 11(1):107-112

15. Tian Y, Zhang C, Guo M (2015) Comparative analysis of amaryllidaceae alkaloids from three lycoris species. Molecules 20:21854-21869

16. Sugiura J, Noguchi S, Nakajima M (2015) Application of coagulant in the analysis of lycorine and galanthamine in processed foods. Food Hygiene Saf Sci 56:108-113

17. Andrade-Eiroa A, Canle M, Leroy-Cancellieri V, Cerdà V (2015) Solid phase extraction of organic compounds: a critical review. Part I. Trends Anal Chem 80:641-654

18. Sugiura J, Nakajima M (2017) Simultaneous determination of nine preservatives in food by liquid chromatography with the aid of coagulant in the clean-up process. Food Additi Contam Part A 34:695-704

19. Gałuszka A, Migaszewski Z, Namieśnik J (2013) The 12 principles of green analytical chemistry and the SIGNIFICANCE mnemonic of green analytical practices. Trends Anal Chem 50:78-84

20. Ozeki S (1964) Studies on the Alkaloids of Zephyranthes candida Herb I. Isol Bases. Yakugaku zasshi 84:1194-1197

21. Meloun M, Bordovska S, Galla L (2010) The thermodynamic dissociation constants of clotrimazole, terbinafine $\mathrm{HCL}$, acetylsalicylic acid, salicylic acid, and galanthamine by the nonlinear regression of multiwavelength spectrophotometric $\mathrm{pH}$-titration data. SRX Pharmacol 2010:Article ID 527013

22. Kretzing S, Abraham G, Seiwert B, Ungemach F, Krügel U, Regenthal R (2011) Dose-dependent emetic effects of the Amaryllidaceous alkaloid lycorine in beagle dogs. Toxicon 57:117-124

23. Meloun M, Andrade J, Pigni N, Torras-Claveria L, Berkov S, Codina C, Viladomat F, Bastida J (2012) Bioactive alkaloid extracts from Narcissus broussonetii: mass spectral studies. J Pharm Biomed Anal 70:13-25

Publisher's Note Springer Nature remains neutral with regard to jurisdictional claims in published maps and institutional affiliations. 